\title{
Weather Forecast Use for Winter Recreation*
}

\author{
Michelle RutTy AND JeAn ANDREy \\ Department of Geography and Environmental Management, University of Waterloo, Waterloo, Ontario, Canada
}

(Manuscript received 8 August 2013, in final form 31 January 2014)

\begin{abstract}
Recent studies have begun to address the importance of weather information for leisure activities. This paper contributes to the understanding of how weather information is sourced, perceived, and used for the discretionary and weather-dependent winter activities of skiing, snowboarding, and snowmobiling. A survey of 1948 Ontario (Canada) skiers/snowboarders and snowmobilers is the empirical basis for the paper, providing insights into how winter recreationists are both similar to and different from the general public with respect to weather information. Results show that virtually all ( $\geq 97 \%)$ skiers/snowboarders and snowmobilers use weather forecasts when planning an outing, which are primarily $(\geq 95 \%)$ sourced through Internet and mobile devices. Skiers/snowboarders and snowmobilers are also highly attentive to rain and freezing rain variables in the forecast, as it negatively affects participation. The results also demonstrate the importance of forecast use for planning travel to snow resorts and snowmobile trails, with poor road conditions likely to result in a postponed or cancelled trip. These findings underscore the differing weather needs of subpopulations, with the need for continued research to examine variations among weather forecast users for context specific decision making.
\end{abstract}

\section{Introduction}

Weather service providers throughout the world provide the public with forecasts and warnings in order to protect life and property through improved decision making. However, effective communication requires an understanding of how intended audiences access and use such information in various decision-making contexts. Recent surveys have found that, in the United States, weather news is one of the most publicly consumed items in the media (Pew Research Center 2008; Wilson 2008). In fact, it has been estimated that 300 billion forecasts are obtained by U.S. adults on an annual basis (Lazo et al. 2009). Despite the widespread consumption of daily weather forecasts, little empirical research has examined how and why the public obtains, perceives, and responds to this information (Lazo et al. 2009; Demuth et al. 2011). Yet such research is fundamental to the design of weather

\footnotetext{
* Supplemental information related to this paper is available at the Journals Online website: http://dx.doi.org/10.1175/WCAS-D13-00052.s1.

Corresponding author address: Michelle Rutty, Department of Geography and Environmental Management, University of Waterloo, 200 University Ave. W., Waterloo, ON N2L 3G1, Canada. E-mail: mkrutty@uwaterloo.ca
}

products and communication strategies, as noted by both scientific organizations (e.g., National Research Council 2006, 2010; National Weather Service 2010; World Meteorological Organization 2013) and individual researchers alike (e.g., Dash and Gladwin 2007; Gladwin et al. 2007; Morss et al. 2008, 2010; Demuth et al. 2011; Stewart et al. 2012).

This study provides insights into how weather information is used by winter recreationists in parts of Ontario, Canada. More specifically, the paper examines the sources of weather information accessed, people's perceptions of the importance and reliability of various forecast variables, and the types of decisions associated with forecast conditions. Previous research, while limited, suggests that weather information is used most for discretionary activities (Lazo et al. 2009; Demuth et al. 2011; Environment Canada 2012a) and that weather information plays an important role in recreation/tourist decisions (Maunder 1969; de Freitas and Wells 1982; Scott et al. 2011). Understanding such decision making requires, at minimum, describing the information used (Stewart et al. 2004; Scott and Lemieux 2010). This study is unique in its focus on discretionary and weatherdependent winter recreation and in its examination of the relationship between weather information and how end users (i.e., recreationists) integrate this information into context-specific decisions. 


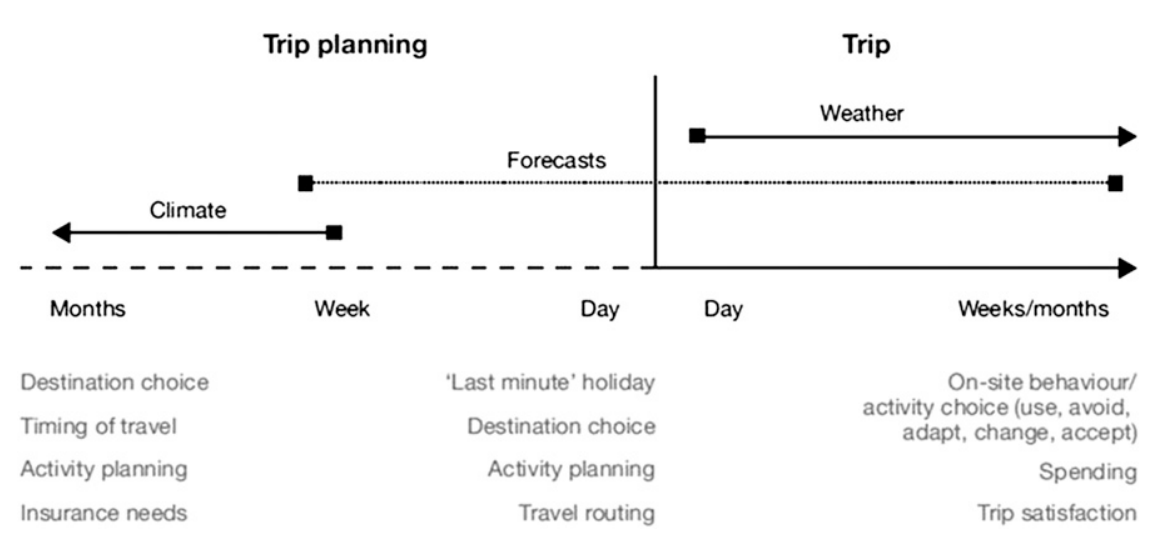

FIG. 1. Weather and climate information for tourist decision making (Scott et al. 2011).

\section{Background}

\section{a. Winter recreation}

Winter sports tourism, including skiing, snowboarding, snowmobiling, ice fishing, and other snow- and icerelated recreational activities, is an important market segment in North America. Such outdoor activities necessitate destination-focused travel to areas with large capital investments in infrastructure. In North America, direct annual revenues associated with skiing and snowboarding are estimated at nearly USD $\$ 7$ billion (National Ski Areas Association 2013; Statistics Canada 2013), with snowmobiling estimated at USD \$34 billion (International Snowmobile Manufacturers Association 2014). The Canadian ski/snowboard and snowmobile share of these revenues is approximately one-fifth and one-quarter, respectively. Although the largest numbers of visits to Canadian snow resorts are to destinations in the provinces of Quebec (33\%) and British Columbia (31\%), the province of Ontario receives $18 \%$ of Canadian skiers and snowboarders (Canadian Ski Council 2009). Ontario is also the primary snow resort destination for Americans who travel north of the border, accounting for more than $60 \%$ of such visits (Canadian Tourism Commission 2006). As for snowmobiling, Ontario has the highest number of registered snowmobiles (149 000) in Canada outside of Quebec (176000; International Snowmobile Manufacturers Association 2014).

The current study focuses on winter recreation in the province of Ontario, Canada. Ontario has a population of 13.5 million ( $38.7 \%$ of Canada's population), with escarpments and open farmland near large urban areas that typically receive abundant snowfall throughout the winter season by virtue of latitude and/or location to the lee of the Great Lakes. During the 2011/12 winter ski season, Ontario snow resorts generated an estimated CAD \$228 million in revenue (Ontario Snow Resorts Association 2013). During the same season, direct expenditure generated by snowmobiling in the province was estimated at over CAD $\$ 1$ billion (Ontario Federation of Snowmobile Clubs 2013).

Tourism/tourists and recreation/recreationalists are generally seen as a set of interrelated and overlapping concepts, with many (contested) definitions (Hall 2006). These concepts are often used interchangeably; tourism embraces the concept of recreation in that it is the practice of traveling for recreation, while recreation can be defined as an activity in which individuals voluntarily engage for personal satisfaction or pleasure (de Freitas 2003). More specifically, recreation is often defined as any pursuit engaged upon during leisure time, except for those in which people are normally highly committed (e.g., shopping, house repairs, overtime, religion, and politics), whereas tourism is generally defined based on a duration of time (e.g., day trip versus an overnight trip) and/or distance (e.g., proximity to destination). The United Nations World Tourism Organization (UNWTO 2013) defines a tourist as someone who takes a trip with an overnight stay, while the Organization for Cooperation and Development (OECD 2013) also stipulates that a tourist is one who travels outside of their country of residence for at least one overnight stay. Some government authorities also include day-trippers in their tourism data, including the Ontario Ministry of Tourism Culture and Sport (2011), which defines a tourist as someone traveling $40 \mathrm{~km}$ one way from home. Therefore, distinguishing between recreationalists and tourists is challenging given that these two concepts change meaning depending on the context of analysis, purpose, and use. In this vein, both terms are used interchangeably here.

\section{b. Weather information for tourism and recreation}

Weather and climate have broad significance to tourist decision making. Figure 1 conceptualizes the influence of different types of weather and climate information 
(historical, forecasts, nowcasts) on tourist decision making, from the trip planning to the travel phase to the destination itself. While there have been few evaluations of weather and climate services for tourists (Scott et al. 2011), the limited available studies consistently indicate widespread use in holiday planning. For example, in Britain, tourism and recreation users were shown to generate the largest demand from automated telephone and weather services (Smith 1993). Gamble and Leonard (2005) found that $95 \%$ of coastal recreationists in North Carolina indicated knowledge of weather conditions as important for making activity and planning decisions. A survey of German outbound tourists found $73 \%$ informed themselves about the climate of their destination and $42 \%$ did so prior to booking their travel (Hamilton and Lau 2005). A similar survey of Northern European travelers to the Mediterranean region found $86 \%$ reviewed information on their destination's climate, with $81 \%$ doing so before making any travel reservations (Rutty and Scott 2010). Over 60\% of visitors to Martinique gathered weather information on the Internet prior to their trip, with an additional $37 \%$ obtaining weather information from television, guidebooks, travel brochures, and travel agencies (Hübner and Gössling 2012).

The importance of weather information for recreational activities has also been noted by the meteorological community, based on various publications related to a U.S. national public survey. Lazo et al. (2009) found that respondents use weather forecasts more for discretionary activities (e.g., planning weekend activities) than for those activities that are not easily changed (e.g., work-related activities). Demuth et al. (2011) found that a primary driver behind how often people obtain forecasts is linked to forecast use, with planning leisure activities resulting in the highest frequency of weather forecast use. In a Canadian context, a national weather service survey by Environment Canada (2012a) similarly revealed that planning outdoor recreation activities was the most popular reason for using weather forecast information, with a high diversity in forecast sources. These studies suggest that weather information is likely to be highly sought and duly considered by winter recreationists, given the discretionary and weather-dependent nature of these activities, and also that different winter recreation subsets may be more or less responsive to different weather variables and conditions.

Literature in the multidisciplinary field of transportation also explores the public's response to weather forecasts and actual weather conditions, often using a theoretical lens that centers on risk compensation during adverse weather conditions. Some drivers cancel or defer trips or change travel modes in consideration of weather forecasts. Studies suggest very low levels of auto trip cancellation during rainfall and light snow, but often substantial reductions during winter storms (Knapp 2001; Kilpeläinen and Summala 2007). Other adjustments, including increased driver attention and vehicle headway (Andrey and Knapper 2003), and reductions in travel speeds (Unrau and Andrey 2006) also occur, although these responses do not alter drivers' exposure to weather hazards per se, but rather ameliorate the risks associated with travel in less-than-ideal driving conditions. Consistent with the literature on weather information use generally, studies related to transportation also indicate that discretionary travel is the most sensitive to weather (Cools et al. 2010a,b).

\section{Methods}

This paper examines the sources, perceptions, and uses of weather forecasts associated with winter recreation at snow resorts and snowmobile trails. The study is based on primary data collected through online surveys. The survey instrument used for snow-resort recreationists was essentially the same as that used for snowmobilers, with some tailoring of questions to reflect the different activity types. The draft survey instruments were peer-reviewed for content and clarity, with revised versions of the surveys launched online via SurveyMonkey in November 2012. After approximately 100 survey responses, the database was checked to confirm survey functionality and data quality, with data collection continuing thereafter for a 1-month period. Participants were recruited through an electronic mailing list provided by the Ontario Snow Resort Association and the Ontario Federation of Snowmobile Clubs, as well as through pamphlets distributed during industry tradeshows. The surveys were distributed in English and were limited to one survey per Internet Protocol (IP) address. A total of 2734 surveys were received. Respondents who did not reside in Ontario (487), answered five questions or less (146), did not consent to participate or were underage (83), or had never visited an Ontario snow resort or snowmobile trail (70) were removed from the sample and were not included in the data analysis. As such, there was an effective survey sample of 1948. From this, 1010 (52\%) were skiers and/or snowboarders and 938 $(48 \%)$ were snowmobilers. Based on these sample sizes, the reported percentages can be interpreted as being accurate to within $\pm 3 \%, 95$ times out of 100 .

To enable comparison across previous studies, the surveys incorporated some questions posed by Lazo et al. (2009) and Morss et al. (2008) (i.e., Q14-Q19), as well as Cools et al. (2010b) (i.e., Q22 and Q23) (see supplemental material). To inventory the weather information 


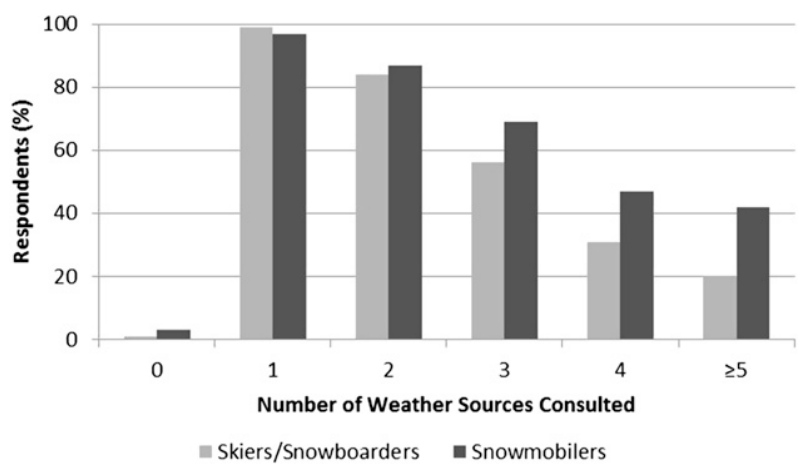

FIG. 2. Number of weather sources consulted when planning a ski/ snowboarding or snowmobiling outing $(n=1948)$.

sources used by winter recreationists, respondents were asked to select all of the sources they utilize when planning an outing. Next, the survey explored how these forecasts are perceived, including an examination of individuals' stated importance of 18 different forecast variables (list provided) when planning an outing, as well as their confidence in weather forecast information for three varying lead times (1, 3, and 5 days in advance). In terms of the use of weather information, respondents were asked about their reasons for accessing weather information when planning an outing to a snow resort or snowmobile trail, as well as their use of weather forecasts in decisions related to skiing/snowboarding or snowmobiling activities. The full survey is available from the authors, with a listing of those questions analyzed in this paper provided in the supplemental material.

The analysis focuses on summary statistics related to the above themes. Statistical tests (difference of means tests, difference of proportion test, and chi-squared analysis) were used to compare response patterns for skiers/ snowboarders versus snowmobilers. Response categories noted with an asterisk indicate a statistically significant difference at the level of 0.05 .

\section{Results}

\section{a. Respondent profiles}

Overall, the two samples are dissimilar in their gender composition, but similar in terms of age profile and engagement in winter recreational activities. In terms of gender, there are differences between the skier/ snowboarder sample and the snowmobile sample, with slightly more females $(60 \%)$ in the skier/snowboarder set and primarily males (85\%) in the snowmobile sample. The ski sample is only slightly younger than the snowmobile sample, with $9 \%$ and $3 \%$ between the ages of 18 and 25 years, respectively, followed by $10 \%$ and $12 \%$ between 26 and 35 years old, $30 \%$ and $27 \%$ between 36 and 45 years

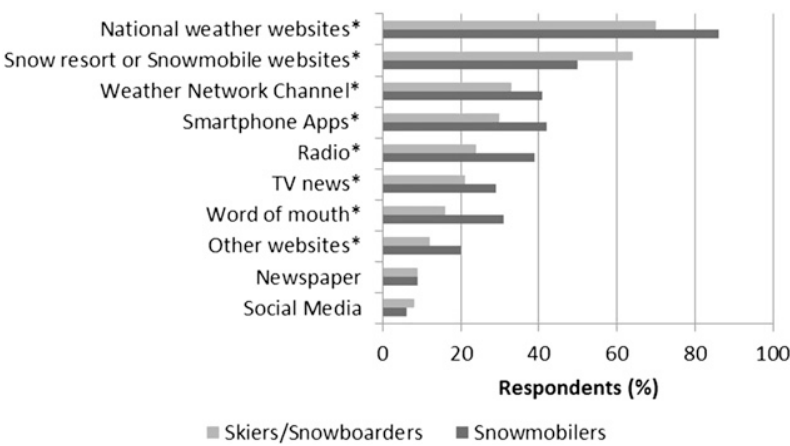

FIG. 3. Weather forecast sources consulted when planning a ski/ snowboarding or snowmobiling outing $(n=1948)$.

old, $35 \%$ and $38 \%$ between 46 and 55 years old, and $15 \%$ and $18 \%$ at 56 years or older. The samples in both surveys are highly experienced, with $77 \%$ of skiers/snowboarders and $71 \%$ of snowmobilers having 10 or more years of experience in their respective activity. Respondents are also highly active in other winter activities, with $80 \%$ of skiers/snowboarders and snowmobilers participating in activities such as skating, ice fishing, snowshoeing, snow tubing, and winter festivals. There is also some crossover between the activities on which the two sample groups are based, with $32 \%$ of snowmobilers participating in skiing and/or snowboarding, and $13 \%$ of skiers/snowboarders participating in snowmobiling.

\section{b. Accessing weather information (sources)}

As illustrated in Fig. 2, almost all skiers/snowboarders $(>99 \%)$ and snowmobilers ( $97 \%)$ check or listen to one weather forecast source when planning an outing to a snow resort or snowmobile trail. On average, skiers/ snowboarders gather weather information from three sources and snowmobilers from four sources. Figure 3 details the variety of sources consulted. Two sources are utilized the most, these being national weather web pages (e.g., Environment Canada and the Weather Network) and snow resorts or snowmobile club web pages. Snowmobilers rely more on the former source than skiers/ snowboarders ( $86 \%$ and $70 \%$, respectively). Almost half of the skiers/snowboarders (47\%) and snowmobilers (44\%) obtain weather forecasts from both of these sources.

The importance of Internet and mobile sources for weather information is also evident, with $99 \%$ of skiers/ snowboarders and $95 \%$ of snowmobilers gathering information in these ways. This includes the use of smartphone applications and social media by skiers/ snowboarders (38\%) and snowmobilers (48\%), in addition to national weather web pages and snow resort or snowmobile club web pages. While traditional sources of communication are used less frequently, television 


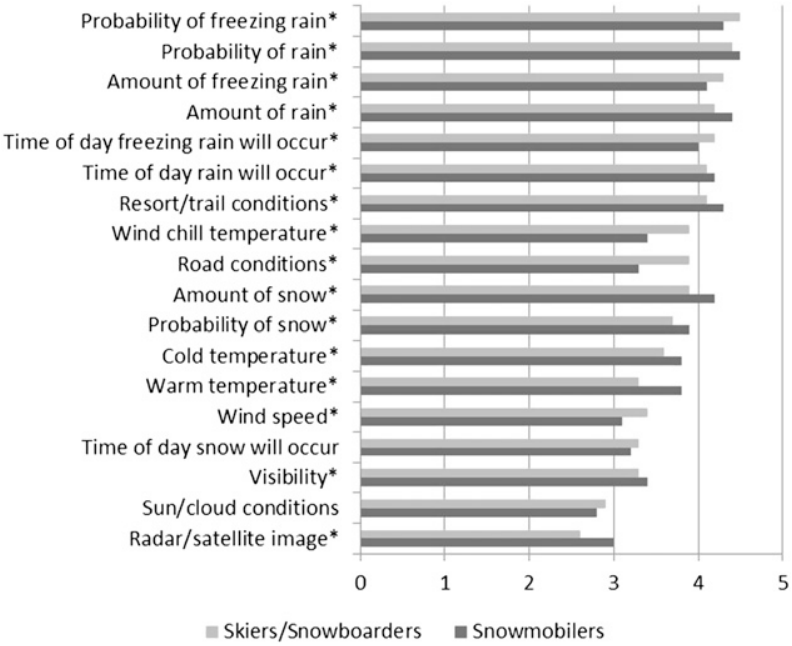

FIG. 4. Importance of weather forecast attributes when planning a ski/snowboarding or snowmobiling outing $(n=1948)$.

remains an importance source, with $54 \%$ of skiers/ snowboarders and $70 \%$ of snowmobilers obtaining forecast information from TV weather stations or TV news. More than one-third of snowmobilers also use the radio as a source of weather information $(38 \%)$, with newspapers sourced the least by both sample groups $(\leq 8 \%)$. Less than $1 \%$ of respondents rely exclusively on traditional sources for weather forecast information.

\section{c. Perceptions}

To better understand how weather forecast information is perceived, respondents were asked to rate the importance of 18 forecast variables when planning a trip to a snow resort or snowmobile trail from one (extremely unimportant) to five (extremely important). More than one-third of the forecast components were rated by the respondents as being very important (4) or extremely important (5). This is illustrated by the mean scores provided in the list below, as well as summarized in Fig. 4, which itemizes each forecast component from the highest to lowest mean score.

Of particular importance to skiers and snowboarders is information on the probability and the amount of precipitation. The probability of rain and the probability of freezing rain were rated the highest and second highest by both skiers/snowboarders (4.4 and 4.5) and snowmobilers (4.5 and 4.3). Almost all respondents ( $\geq 80 \%$ ) stated the probability of rain and the probability of freezing rain are important or extremely important forecast variables, with $\leq 6 \%$ stating these forecast variables as unimportant or extremely unimportant. The amount of rain and the amount of freezing rain were also rated highly by both skiers/snowboarders (4.2 and 4.3) and snowmobilers (4.4 and 4.1). More than three-quarters of respondents $(\geq 75 \%)$ stated the amount of rain and the amount of freezing rain were important or extremely important weather forecast variables, with $\leq 9 \%$ stating these forecast variables as unimportant or extremely unimportant.

The timing of the rain and the timing of freezing rain are also important, with mean importance scores of 4.1 and 4.2 by skiers/snowmobilers and 4.2 and 4.0 for snowmobilers. A large majority of respondents ( $\geq 70 \%)$ stated these weather forecast variables were important or extremely important, with $\leq 13 \%$ stating these variables as unimportant or extremely unimportant.

Information on snow, including the probability of snow, amount of snow, and the timing of snow, are somewhat less important to both sample groups, with the three snow variables having mean importance scores between 3.2 and 4.2, with snow information being slightly more important to snowmobilers than to skiers/snowboarders. Nevertheless, $\geq 61 \%$ of respondents stated the probability of snow and the amount of snow as either important or extremely important, and $\leq 15 \%$ stated these variables as unimportant or extremely unimportant. Furthermore, $\geq 45 \%$ of respondents stated that the timing of snow was important or extremely important to them, with $\leq 27 \%$ stating this forecast variable was unimportant or extremely unimportant.

Temperature attributes, including cold, warm, and wind chill ${ }^{1}$ temperature, are also somewhat less important than the occurrence of rain or freezing rain, with mean importance scores between 3.3 and 3.9 across both sample groups. Given typical differences in clothing for various outdoor sports, it is not surprising that skier/ snowboarders rated the importance of cold temperature (including wind chill) higher than snowmobilers; snowmobilers are able to better protect themselves from low temperatures through heavier apparel and even heated helmets and clothing (e.g., gloves, pants, vests, and jackets) that can be plugged into the $12-\mathrm{V}$ electrical system in their sleds. By contrast, snowmobilers expressed more concern about warm temperatures, which not only affects trail conditions, but can also strain snowmobile engines. The majority of snowmobilers $(64 \%)$ rated warm temperatures as important or extremely important, compared to $45 \%$ of skiers/snowboarders. Conversely, wind chill temperatures were important or extremely important to $68 \%$ of skiers/snowboarders compared to $51 \%$ snowmobilers. For all three temperature attributes,

\footnotetext{
${ }^{1}$ Canada's wind chill index is an index that is "expressed in temperature-like units by equating the current outdoor conditions to an equivalent temperature with no winds" (Environment Canada 2012b).
} 


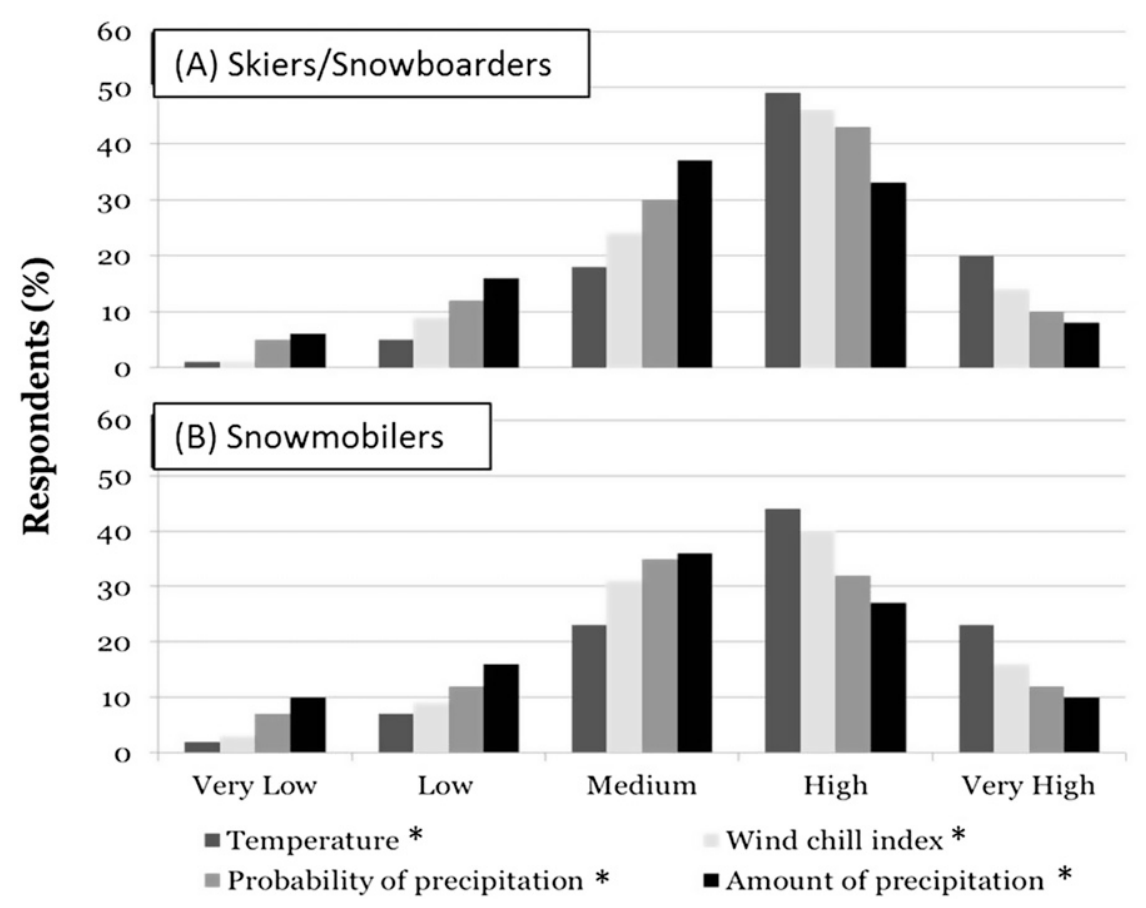

FIG. 5. Respondents' confidence in different types of 1-day weather forecast attributes $(n=1948)$.

$\leq 25 \%$ of respondents rated them to be unimportant or extremely unimportant when planning a ski/snowboarding or snowmobile outing.

Sun/cloud conditions and radar/satellite image were the only two components of weather forecasts that received mean importance scores of $\leq 3$ by both groups of recreationists. Radar/satellite image was slightly more important to snowmobilers (38\% stated this variable as important or extremely important) compared to skiers/snowboarders $(24 \%)$. While skiers/snowboarders can gather weather forecast information for a location-specific snow resort, snowmobilers may ride trails for hundreds of kilometers per day, spanning a wide geographical area and passing through numerous communities en route. Radar/satellite image may therefore provide more accessible and comprehensive weather information. For sun/cloud conditions, $29 \%$ of respondents from both sample groups stated it to be important or extremely important.

To further illuminate how forecasts are perceived, Figs. 5-7 summarize the confidence that skiers/ snowboarders and snowmobilers have in weather forecasts that are given 1,3 , and 5 days in advance. As shown, respondents have more confidence in forecasts with shorter lead times and in the forecast variables, temperature, and wind chill. For a forecast given 1 day in advance, a majority of respondents have high or very high confidence in both temperature and wind chill and either medium or high confidence in the probability of precipitation and the amount of precipitation. For forecasts given three days in advance, confidence levels are considerably lower. For temperature and wind chill, respectively, only $22 \%$ and $13 \%$ report high or very high confidence in forecast conditions, with approximately one-third indicating medium confidence and the remainder rating their confidence as low or very low. For the two precipitation variables, more than one-half of the respondents have low or very low confidence in 3-day forecasts. For 5-day forecasts, more than three-quarters of respondents have low or very low confidence in all four weather variables. Both skiers/snowboarders and snowmobilers have similar levels of confidence both for forecasts of varying lead times and for the different weather variables.

\section{d. Uses}

To examine how skiers/snowboarders and snowmobilers use forecasts when planning an outing, respondents were asked to indicate how frequently they access weather information for different purposes. In each case, they were asked to provide a rating from one (never) to five (always). As illustrated in Fig. 8, more than $70 \%$ of respondents in both sample groups indicate that they always use the forecast to simply know what the weather will be like. By comparison, when asked about three other specific uses-planning how to dress oneself or one's children, planning travel for a winter 


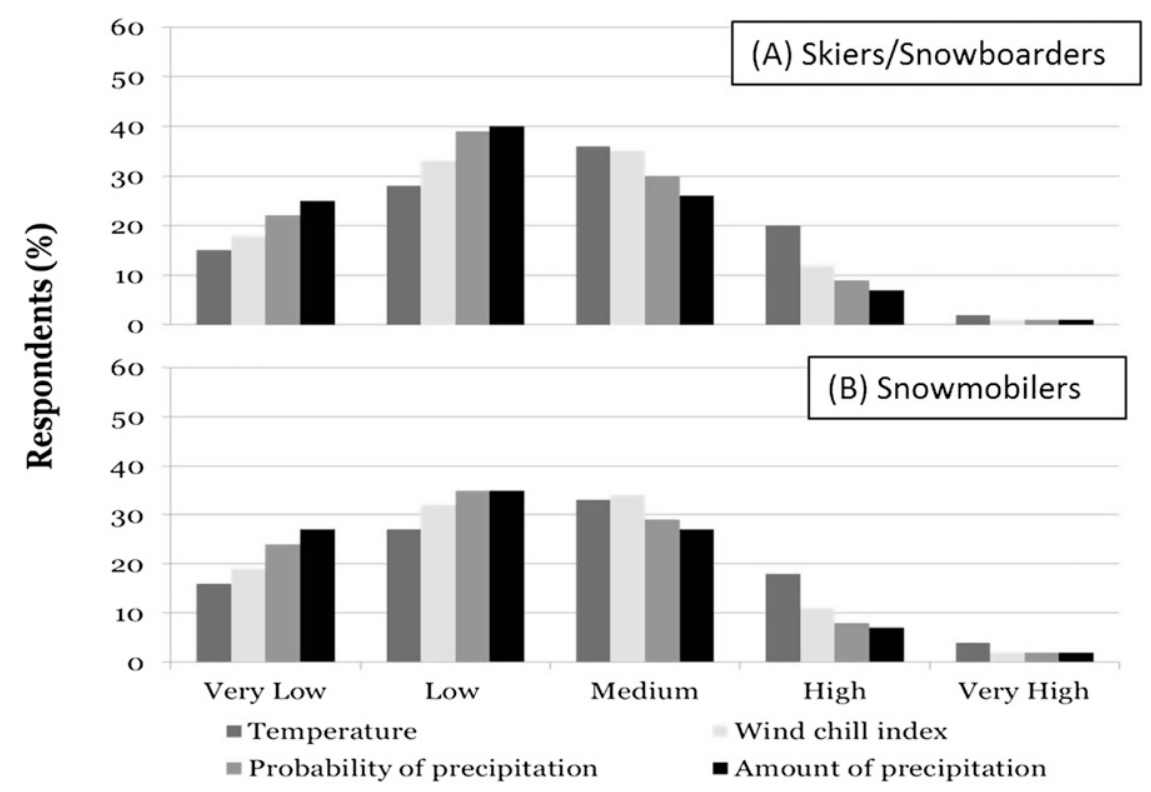

FIG. 6. Respondents' confidence in different types of 3-day weather forecast attributes $(n=1948)$.

recreational outing, or deciding the length of an outingthe frequency with which people access weather information is lower overall. More specifically, for the query about clothing, $67 \%$ of skiers/snowboarders and $51 \%$ of snowmobilers state that they always use the forecast to help with their decision making, with an additional $19 \%$ and $21 \%$, respectively, using the forecast more than half the time. It is possible that skiers/ snowboarders use weather forecasts more when deciding on how to dress themselves or their children because they are more directly exposed to the outdoor thermal conditions and rely on the layering of clothing to keep warm during winter weather conditions. Conversely, snowmobilers' attire (especially facial coverage) is typically warmer,

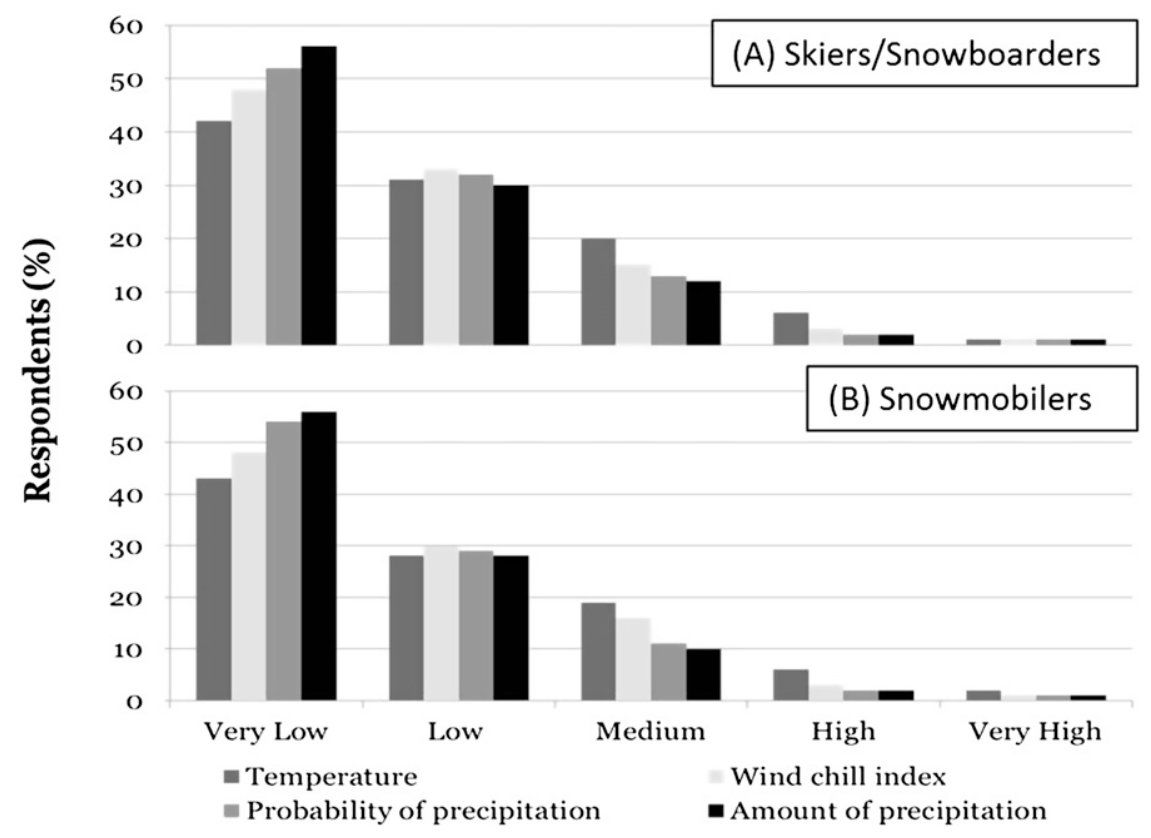

FIG. 7. Respondents' confidence in different types of 5-day weather forecast attributes $(n=1948)$. 


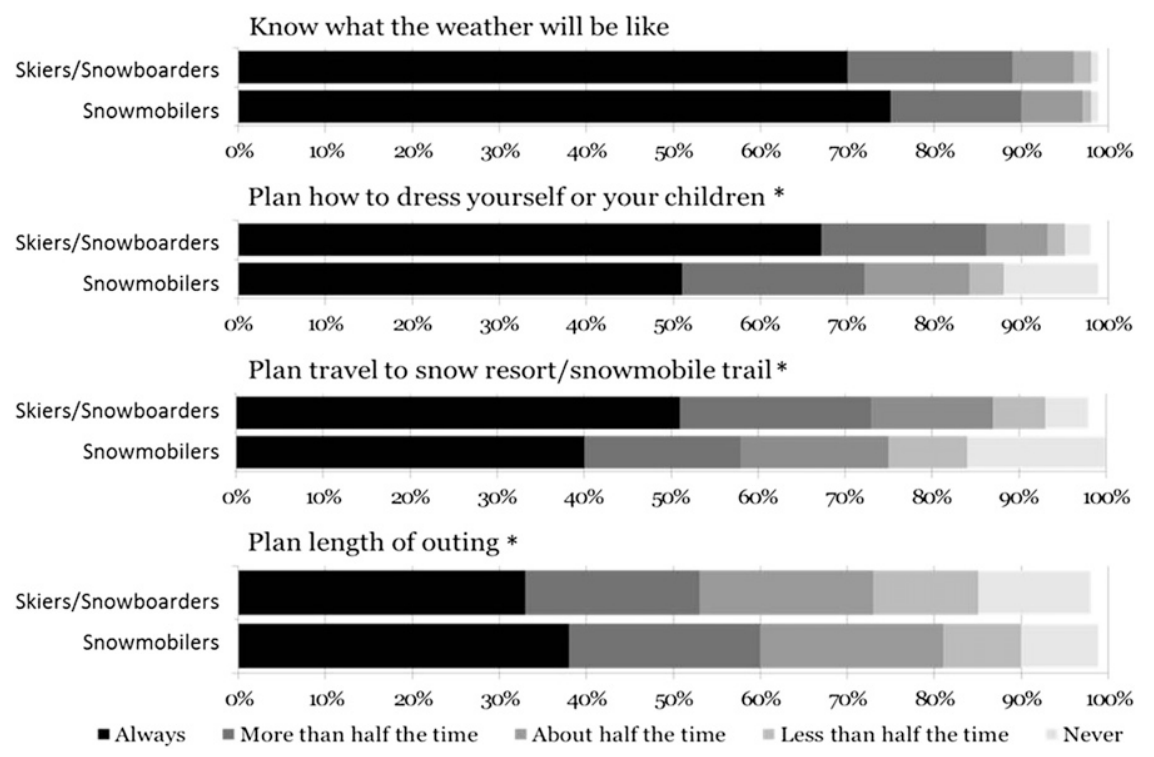

FIG. 8. Respondents' use of weather forecast when planning a ski/snowboarding or snowmobiling outing $(n=1948)$.

with the additional option to electrically heat their apparel, minimizing concern over thermal discomfort.

For planning travel, skiers/snowboarders use the forecast more than snowmobilers, with $73 \%$ of skiers/ snowboarders using the forecast always or more than half the time when traveling to a snow resort, compared to $58 \%$ for snowmobilers traveling to a snowmobile trail. These findings may be associated with differences in travel distances between the two groups, but available data provide information only on whether the travel distance exceeds $40 \mathrm{~km}$ and are not sufficient to explore this in depth. Rather, the survey data indicate that the vast majority of respondents in both sample groups (90\% of skiers/snowboarders and $81 \%$ of snowmobilers) typically do travel more than $40 \mathrm{~km}$ from their home in order to participate in activities at snow resorts or on snowmobile trails.

While weather forecasts are used the least when deciding on the length of an outing, the majority of both skiers/snowboarders $(53 \%)$ and snowmobilers $(60 \%)$ nevertheless use the forecast always or more than half the time. Length of outing may be more important to snowmobilers because their activity is limited to daylight hours, whereas skiers/snowboarders have greater flexibility, with most Ontario snow resorts offering nighttime skiing until 2200 LT.

To better understand how weather forecasts affect the decision to participate in a winter recreational activity, respondents were asked which, if any, weather forecast variables would deter them from going skiing/snowboarding or snowmobiling. The survey results show that $84 \%$ of skiers/snowboarders and $81 \%$ of snowmobilers would be deterred from going to a snow resort or snowmobile trail as a result of a weather forecast. As illustrated in Fig. 9, of those respondents who would be deterred, rain and freezing rain were the greatest deterrents for both skiers/snowboarders ( $89 \%$ and $93 \%$ ) and snowmobilers $(96 \%$ and $81 \%)$. The majority of skiers/snowboarders $(56 \%)$ would also be deterred by high wind chill and a majority of snowmobilers (61\%) would be deterred by warm temperatures. Few respondents in both sample groups would be deterred by sun/cloud conditions $(<2 \%)$ or by other forecast variables including lack of snow, high winds, and fog $(<4 \%)$.

To better understand the influence of temperature on the decision to participate in a winter recreational

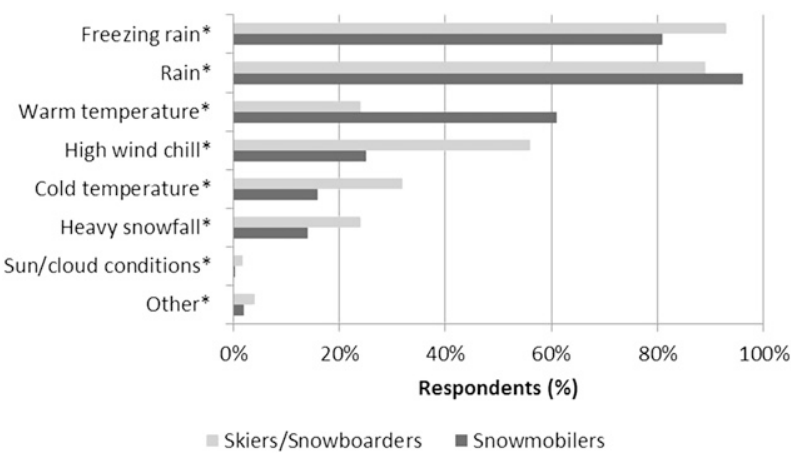

FIG. 9. Weather forecast variables that would deter skiers/ snowboarders or snowmobilers from planning an outing $(n=1948)$. 
activity, respondents were also asked how likely they are to go ski/snowboarding or snowmobiling under different temperature forecast scenarios (Fig. 10). Between $0^{\circ} \mathrm{C}$ $\left(32^{\circ} \mathrm{F}\right)$ and $-15^{\circ} \mathrm{C}\left(5^{\circ} \mathrm{F}\right)$, the majority $(\geq 70 \%)$ of respondents stated they are likely or very likely to go ski/ snowboarding or snowmobiling. At $-5^{\circ} \mathrm{C}\left(23^{\circ} \mathrm{F}\right)$, the largest share of ski/snowboarders $(96 \%)$ and snowmobilers $(92 \%)$ stated they would be likely or very likely to participate in their respective outdoor activity. At $-20^{\circ} \mathrm{C}\left(-4^{\circ} \mathrm{F}\right), 75 \%$ of snowmobilers stated they are likely or very likely to go snowmobiling, compared to less than half of skiers/snowboarders $(41 \%)$. Between $-25^{\circ} \mathrm{C}\left(-13^{\circ} \mathrm{F}\right)$ and $-30^{\circ} \mathrm{C}\left(-22^{\circ} \mathrm{F}\right), 20 \%$ and $12 \%$ of skiers/snowboarders are likely or very likely to go ski/ snowboarding, which is much lower than the $49 \%$ and $33 \%$ of snowmobilers that would plan an outing at these two temperatures. The opposite was recorded at warmer temperatures, with the majority (62\%) of ski/snowboarders likely or very likely to plan an outing at $5^{\circ} \mathrm{C}\left(41^{\circ} \mathrm{F}\right)$, compared to the majority $(53 \%)$ of snowmobilers who are unlikely or very unlikely to plan an outing.

The influence of road conditions on travel decisions was also examined. Respondents were asked whether or not they would alter travel plans to a snow resort or snowmobile trail (i.e., travel to a snowmobile staging area) if the roads had blowing snow and/or poor visibility or if the roads were icy/snow packed. A majority of skiers/snowboarders responded that they would alter plans in both road scenarios $(66 \%$ and $53 \%$, respectively), while a majority of snowmobilers would not alter plans for either road scenario $(40 \%$ and $26 \%$, respectively). As previously noted, these findings may be attributable to differences in travel distances. Skiers/ snowboarders have fewer options in terms of available snow resorts and may subsequently have to travel farther distances to participate in their outdoor activity. Conversely, there are numerous staging areas available to snowmobilers, presumably at varying travel distances. Snowmobilers may therefore have the option of selecting snowmobile trails that are closer to home, minimizing travel time on roads with poor forecasted conditions.

Figures 11 and 12 further detail traveler adjustments due to poor road conditions. As shown here, the most common decision for both groups is to change time of departure. As well, a majority of skiers/snowboarders indicate that they are likely to cancel their trip because of blowing snow and/or poor visibility $(61 \%)$ or because of icy/snow-packed roads (63\%) and are less likely to change their trip destination (i.e., travel to a different snow resort) because of either road scenario ( $24 \%$ and $25 \%$, respectively). Conversely, snowmobilers are less likely to cancel their outing because of blowing snow and/or poor visibility ( $42 \%$ ) or because of icy/snow-packed roads $(40 \%)$ and are more likely to change their trip destination (i.e., select a different staging area; $49 \%$ and $42 \%$ ). Finally, when respondents were prompted as to whether they are likely to change travel modes as a result of the weather, less than $10 \%$ of respondents in both sample groups indicated that they are likely to make such a change.

\section{Conclusions and interpretations}

It was anticipated that skiers/snowboarders and snowmobilers frequently access weather forecasts, and the results bear this out. Based on the survey data, virtually all ( $\geq 97 \%)$ skiers, snowboarders, and snowmobilers access weather forecasts when planning an outing. Accordingly, we conclude that winter recreationists are heavy users of weather forecasts.

With respect to information sources, the current study highlights the multiplicity of venues that are used to access forecast information, a conclusion that is consistent with the findings from previous surveys in both the United States (Lazo et al. 2009) and Canada (Environment Canada 2012a). However, skiers/ snowboarders and snowmobilers appear to have less variability in their sources than what occurs for the public at large, with a majority relying on two primary sources for their weather information, namely, national weather web pages and snow resort or snowmobile organization web pages. In previous public surveys, no dominant source(s) emerged. Moreover, almost all skiers/snowboarders and snowmobilers rely on Internet and mobile communication as a source of weather information, with a high usage of smart phone applications ( $30 \%$ and $42 \%$, respectively), leading to the conclusion that winter recreationists are relying on primarily nontraditional outlets for weather information. This finding is in direct contrast to the other available studies that found that traditional sources (e.g., television and radio) are accessed most frequently (Lazo et al. 2009; Demuth et al. 2011; Environment Canada 2012a). Therefore, a collectively strong source preference is evident for skiers/ snowboarders and snowmobilers, with this sample's forecast-acquisition behavior accentuating the general importance of Internet and mobile communication and the specific importance of two primary web pages. This finding may be related, in part, to timing of this study vis-à-vis earlier studies, given the rapidly changing nature of phone technologies, but it has implications for public and private weather service providers in product and service delivery.

The results confirm previous studies' conclusions about the importance of weather forecasts for leisure activities (e.g., Lazo et al. 2009; Demuth et al. 2011; Environment Canada 2012a) and provide new insights 


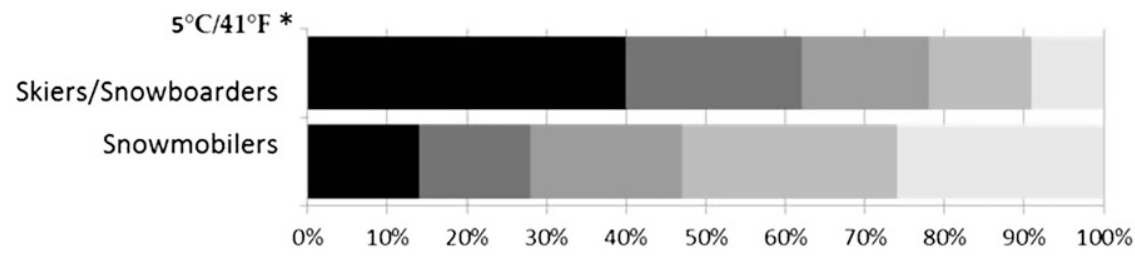

Skiers/Snowboarders

Snowmobilers

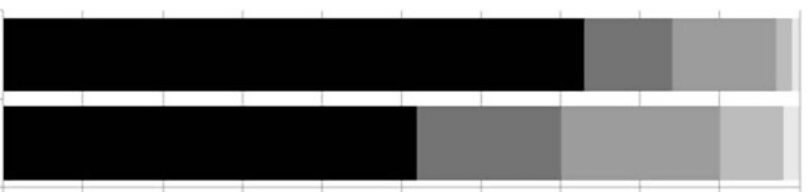

$-5^{\circ} \mathrm{C} / 23^{\circ} \mathrm{F}$

Skiers/Snowboarders

Snowmobilers
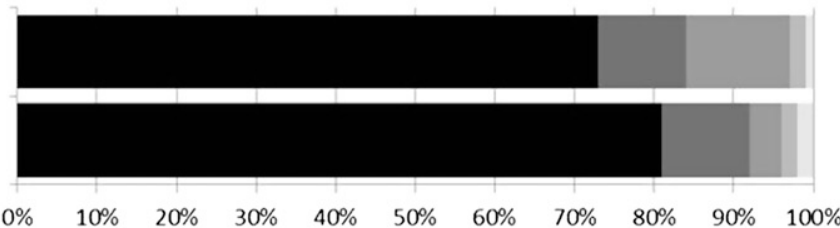

$-10^{\circ} \mathrm{C} / 14^{\circ} \mathrm{F} *$

Skiers/Snowboarders

Snowmobilers

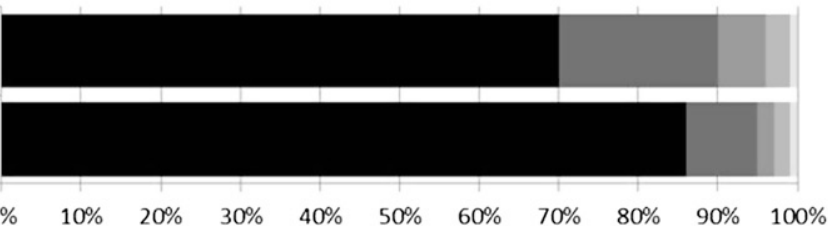

$-15^{\circ} \mathrm{C} / 5^{\circ} \mathrm{F}^{*}$

Skiers/Snowboarders

Snowmobilers
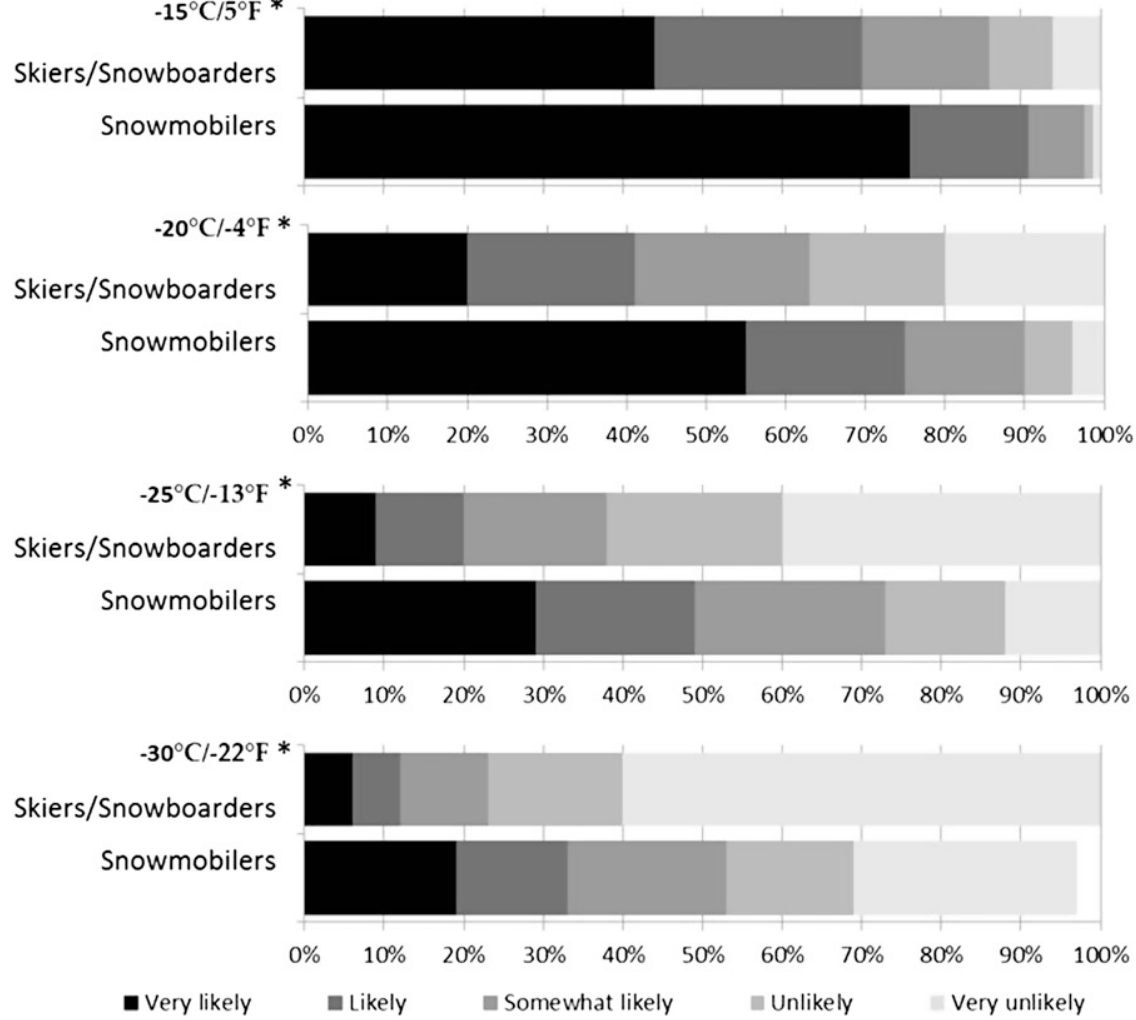

FIG. 10. Likelihood of planning a ski/snowboarding or snowmobiling outing under various temperature forecast scenarios $(n=1948)$. 


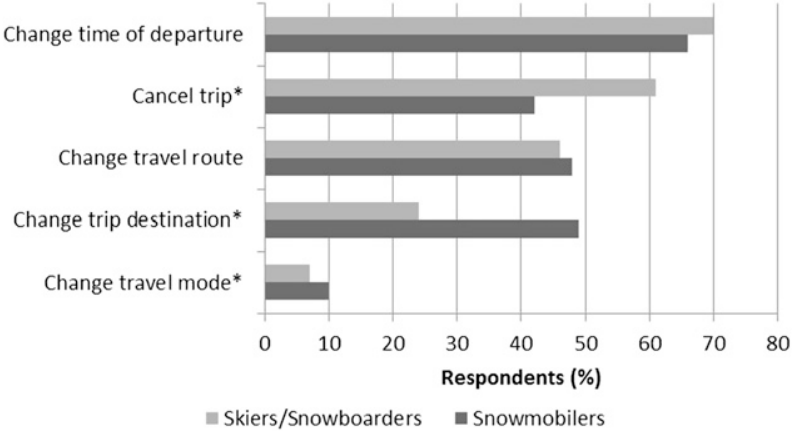

FIG. 11. Respondents' changes in travel behavior due to forecasted blowing snow and/or poor visibility en route to snow resort or snowmobile trail (i.e., staging area; $n=669$ ).

into the importance of specific weather conditions for winter recreational activities. In the current study, forecasts of precipitation, especially rainfall and freezing rain, were found to be particularly important for skiers, snowboarders, and snowmobilers. Although Lazo et al. (2009) also found that the U.S. public rated precipitation parameters as the most important components of a weather forecast, a comparatively smaller proportion of their sample rated these parameters as being extremely important. The importance of rain and freezing rain in weather forecasts is likely because of their direct and negative effect on winter recreation. Not only do such occurrences degrade the conditions needed to participate in skiing/snowboarding and snowmobiling, but they also compromise comfort and personal safety while on the hills and trails. As a result, winter recreationists are highly sensitive to conditions with precipitation. Accordingly, it is likely that weather forecasts with high spatial and temporal precipitation information will likely be more important and potentially more valuable to this subset of the population.

In line with the literature (e.g., Morss et al. 2008), the results from this study indicate that people's confidence in weather forecasts decreases with increasing forecast lead times and varies by weather parameter. Winter recreationists have the highest confidence in temperature forecasts, followed by probability of precipitation, wind chill temperatures, and amount of precipitation. In agreement with Lazo et al. (2009), these findings suggest that people do have well-formed judgments about weather forecasts and the inherent uncertainty implied with forecasts of greater lead times. Respondents may also have higher confidence in temperature forecasts compared to probability of precipitation because of the subjective nature of the former variable. For example, people can feel whether it is raining or not raining and thereby validate or disprove the forecast, whereas people may not be able to feel whether the temperature is

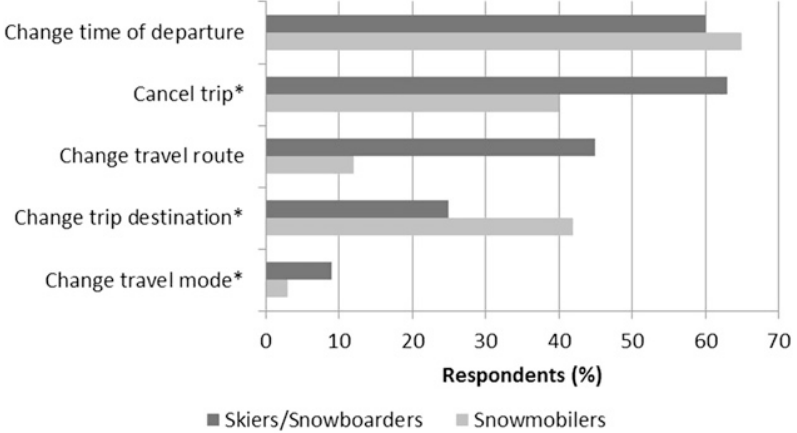

FIG. 12. Respondent's changes in travel behavior due to forecasted icy/snow-packed roads en route to snow resort or snowmobile trail (i.e., staging area; $n=534$ ).

varying from the forecasted conditions, minimizing the ability to validate or disprove the forecast. Rain is also a more absolute measure in that it is either raining or not raining, whereas temperature occurs along a scale and is always present.

The results from this study also show the importance of forecast use in decision making, with more than $80 \%$ of respondents stating that the weather forecast could deter them from participating in winter recreational activities. Interestingly, winter recreationists who would be deterred from participating based on a weather forecast rated the importance of all 18 weather forecast variables an average of 0.5 higher than those respondents who would not be deterred by the forecasts; the former group also has a higher confidence in weather forecasts for all three lead times. Weather information can therefore represent a double-edged sword because, while accurate forecasts are valuable, inaccurate forecasts can unnecessarily deter visitation to snow resorts and snowmobile trails-a lament often heard among tourism managers and operators (e.g., Scott et al. 2011; Becken and Wilson 2013).

The results from this study also show that weather forecasts are used when deciding whether or not to travel to a snow resort or snowmobile trail. Previous studies have shown that both the type of weather and the nature of activity affect the likelihood of a change in travel behavior. More specifically, discretionary activities (e.g., leisure activities) have been found to be most strongly associated with behavioral adjustments such as postponement or cancelation (e.g., Cools et al. 2010b). Findings from the current study also lead to the conclusion that discretionary travel for winter recreational activities is weather sensitive, with postponement being the most likely travel change overall. Important differences that were found between skiers/snowboarders and snowmobilers pertain to two specific travel adjustments. While the majority of skiers/snowboarders were more 
likely to alter travel plans because of forecasted weather en route to their destination and was more likely to cancel their trip because of the forecast, the majority of snowmobilers would not alter their plans, and those who would were more likely to change the trip destination. A likely explanation is that snowmobilers have access to a greater variety of snowmobile trails, whereas skiers/ snowboarders are more limited in their destinations (Ontario has approximately $35000 \mathrm{~km}$ of groomed snowmobile trails versus 63 snow resorts). It is also possible that snowmobilers have a different orientation to weatherrelated risks compared to skiers/snowboarders. According to sports psychology, there is a relationship between sensation seeking and the particular sport discipline one chooses, which implies progressive levels of risk-taking behavior (e.g., Zuckerman 1979, 1983; Horvath and Zuckerman 1993; Jack and Ronan 1998). Sports can be classified along a continuum based on the associated risks involved, from high risk (i.e., risk of fatal injury) to low risk (i.e., injuries can occur but probability of fatalities is remote). Research conducted in the area of sensation seeking and sport participation show that while both skiing/snowboarding and snowmobiling are high risk sports, motor-based sports, including snowmobiling, are higher risk (Chirivella and Martinez 1994; I. Kusyszyn et al. 1974, unpublished manuscript). Such high risk athletes indicate behavioral traits that actively seek unusual sensations of speed, defiance of gravity, and impulsiveness (Zuckerman 1979; Jack and Ronan 1998). These traits may subsequently suppress the desire to alter or cancel travel plans in lieu of poor weather conditions and travel safety precautions. Nevertheless, a large minority of snowmobilers did indicate that they are likely to cancel trips because of forecasted poor road conditions en route to snowmobile trails. These results once again demonstrate that weather matters.

\section{Contributions and future research}

An understanding of how intended audiences access and use weather information in their decisions is a prerequisite for the effective communication of weather forecasts. Recent studies have begun to address this, with findings highlighting the importance of weather information for leisure activities (e.g., Lazo et al. 2009; Demuth et al. 2011; Environment Canada 2012a). Building on this knowledge, this paper provides initial insight into how weather information is perceived and used for leisure activities, with a focus on the discretionary and weather-dependent winter activities of skiing, snowboarding, and snowmobiling. The results confirm the importance of weather forecast use for leisure, while underscoring key differences in how winter recreationists attain and perceive forecasts compared to the general public. Important research findings are that virtually all skiers/snowboarders and snowmobilers attain weather forecasts when planning an outing, which are primarily sourced through the Internet and mobile devices. Skiers/ snowboarders and snowmobilers are highly attentive to specific forecast variables that both affect the quality of their experience during the activity and their travel to snow resorts and snowmobile trails. In many instances, the use of weather information was significantly different ( $\alpha=0.05)$ between skiers/snowboarders and snowmobilers, reflecting differences in outdoor clothing, weather parameters that affect how "good" the conditions are for the particular activity, and flexibility in the choice of location for the outdoor activity. While both groups are heavy users of multiple sources of weather information, skiers/snowboarders access more information from snow resort websites, while snowmobilers are more reliant on national weather services and associated websites and TV channels. Another difference is that skiers are more likely to use weather forecasts to make decisions about clothing and travel, while snowmobilers are more reliant on information in order to plan the length of outings. With respect to forecast variables that would deter people from undertaking travel in order to participate in these recreational activities, skiers/snowboarders put more emphasis on freezing rain, wind chill, cold temperatures, and heavy snow, while snowmobilers give greater consideration to rain and warm temperatures; these findings reflect differences in both clothing and snow conditions necessary for high-quality experiences. Finally, the likelihood of planning or cancelling an outing also differed between the two groups. Skiers/snowboarders expressed less concern about temperatures at/near the freezing point and less tolerance for extreme cold than snowmobilers, and also more readiness to cancel a trip because of poor travel weather. Snowmobilers expressed more readiness to change their intended destination, reducing the probability of event cancellation. These differences underscore the high degree of weather sensitivity of skiers/snowboarders in particular and how both conditions at the hill as well as in the surrounding area are important, as they in turn affect participants' comfort while outside and their perceived experience/safety in traveling to/from the snow resort. At present, outdoor recreationists piece together weather forecasts and observed information from multiple sources in order to meet their particular needs, suggesting that there would be both demand for and value in providing tailored and integrated weather products for the various winter recreationist communities.

Literature on the economic value of weather information is growing (Katz and Murphy 1997; Lazo and Chestnut 2002; Lazo et al. 2009), with recent estimates 
valuing weather forecasts services for U.S. households to be USD\$31.5 billion per annum (Lazo et al. 2009). Recreation and tourism, however, is virtually absent from this literature (Scott et al. 2011). There are no known studies that have explored the financial and nonmarket value of weather forecasts for tourism, nor any studies that have examined tourists' "willingness to pay" for weather information. Consequently, the value of weather information for recreationists and tourists is entirely unknown. As Scott et al. (2011) point out, with over 922 million international tourist arrivals and 8 billion domestic trips, plus tourists' willingness to invest personal time and money into travel, the economic value of weather information is considerable even without any consideration of the value for supply-side operations and marketing. Value estimates based on tourists' stated preferences and uncovering the potential value of weather information for the tourism sector therefore remains a critical area for future inquiry.

As communication technologies advance and become more mobile, the ways in which people access weather information are also likely to change. The public is increasingly relying on Internet and mobile sources for weather information, such as the results found here, enabling instantaneous and real-time weather access. An interesting avenue for future research would be to examine whether the frequency of forecast use (e.g., daily access), and the time(s) of day when people obtain forecasts, increases as a result. Weather information is also becoming more interactive through the rise of social media (e.g., social networks, Internet forums, and blogs). Users are increasingly able to share and exchange weather information in virtual communities and networks. It remains unclear what role changing communication may play in the way users receive and respond to weather forecasts. The increasingly diverse weather sources may overwhelm individuals, with the possibility that such changes could affect the credibility of the weather information users are receiving. To effectively target communication and ensure weather providers are meeting the needs of end users, people's sources, perceptions, and uses of weather information need to be continually examined. While it is important to perform these assessments on the general public, subpopulations can have differing weather needs, as demonstrated here with winter recreationists. As such, new and continued assessments need to be completed for context-specific decision making. Understanding how individuals use and perceive forecasts can substantiate the effectiveness of weather information being communicated to the public and can help identify gaps in these processes. In so doing, we can understand how forecast-acquisition behavior is changing over time and ensure that the knowledge on which weather forecasting decisions are based remains relevant and up to date.

Acknowledgments. This work is supported by Ontario Snow Resorts Association (OSRA), Ontario Federation of Snowmobile Clubs (OFSC), Canadian Ski Council (CSC), and Mitacs Accelerate. Thank you to Bruce Haynes (OSRA), Ron Purchase (OFSC), Tim West (OFSC), Patrick Arkeveld (CSC), and Paul Pinchbeck (Blue Mountain Resorts) for your input and feedback throughout the project. Thank you also to Tania Del Matto of My Sustainable Canada for your continued assistance and inkind contribution towards this project. The views and opinions in this paper are those of the authors.

\section{REFERENCES}

Andrey, J., and C. K. Knapper, 2003: Motorists' perceptions of and responses to weather hazards. Weather and Transportation in Canada, J. Andrey, and C. K. Knapper, Eds., Department of Geography Publication Series, No. 55, University of Waterloo, 95-118.

Becken, S., and J. Wilson, 2013: The impact of weather on tourist travel. Tourism Geogr., 15, 620-639, doi:10.1080/ 14616688.2012.762541.

Canadian Ski Council, 2009: 2008-2009 Canadian skier and snowboarder facts and stats. 26 pp. [Available online at xcski.org/ news $/ 2009 \% 20$ Facts $\% 20$ and $\% 20$ Stats \%20final\%20draft.pdf.]

Canadian Tourism Commission, 2006: U.S. winter outdoor activity participants: A special analysis of the Travel Activities and Motivation Survey (TAMS), 35 pp. [Available online at http:// en-corporate.canada.travel/sites/default/files/pdf/Research/ Product-knowledge/TAMS/US\%20Outdoor\%20Activity/ tams_report_U.S._winter_outdoor.pdf.]

Chirivella, E. C., and L. M. Martinez, 1994: The sensation of risks and motivational tendencies in sports: An empirical study. Pers. Individ. Differ., 16, 777-786, doi:10.1016/0191-8869(94)90219-4.

Cools, M., E. Moons, and G. Wets, 2010a: Assessing the impact of weather on traffic intensity. Wea. Climate Soc., 2, 60-68, doi:10.1175/2009WCAS1014.1.

,-- , and $-2010 \mathrm{~b}$ : Changes in travel behavior in response to weather types. Transp. Res. Rec., 2157, 22-28, doi:10.3141/ 2157-03.

Dash, N., and H. Gladwin, 2007: Evacuation decision making and behavioral responses: Individual and household. Nat. Hazards Rev., 8, 67-77, doi:10.1061/(ASCE)1527-6988(2007)8:3(69).

de Freitas, C. R., 2003: Tourism climatology: Evaluating environmental information for decision making and business planning in the recreation and tourism sector. Int. J. Biometeor., 48, 4554, doi:10.1007/s00484-003-0177-z.

_ , and K. M. Wells, 1982: Reassessment of weather forecast terminology and content. Wea. Climate, 2, 16-22.

Demuth, J. L., J. K. Lazo, and R. E. Morss, 2011: Exploring variations in people's sources, uses, and perceptions of weather forecasts. Wea. Climate Soc., 3, 177-192, doi:10.1175/ 2011WCAS1061.1.

Environment Canada, 2012a: National weather and environmental services survey 2012. Consultant's Rep., Harris/Decima, Ottawa, Ontario, Canada, 139 pp. [Available upon request from 1800-160 Elgin St., Ottawa, ON K2P 2P7, Canada.] 
__ cited 2012b: Canada's wind chill index. [Available online at www.ec.gc.ca/meteo-weather/default.asp?lang $=$ En\&n= 5FBF816A-1.]

Gamble, D. W., and L. A. Leonard, 2005: Coastal climatology products for recreation and tourism end users in southeastern North Carolina. Final Rep., NOAA Coastal Services Center, 49 pp. [Available online at http://secoora.net/documents/ planning/Report-Coastal-Climatology-Product-UNCW.pdf.]

Gladwin, H., J. K. Lazo, B. H. Morrow, W. G. Peacock, and H. E. Willoughby, 2007: Social science research needs for the hurricane forecast and warning system. Nat. Hazards Rev., 8, 87-95, doi:10.1061/(ASCE)1527-6988(2007)8:3(87).

Hall, C. M., 2006: The Geography of Tourism and Recreation: Environment, Place and Space. Routledge, $427 \mathrm{pp}$.

Hamilton, J. M., and M. A. Lau, 2005: The role of climate information in tourist destination choice decision-making. Tourism and Global Environmental Change, S. Gössling and C. M. Hall, Eds., Routledge, 229-250.

Horvath, P., and M. Zuckerman, 1993: Sensation seeking, risk appraisal, and risky behaviour. Pers. Individ. Differ., 14, 41-52, doi:10.1016/0191-8869(93)90173-Z.

Hübner, A., and S. Gössling, 2012: Tourists perceptions of extreme weather events in Martinique. Journal of Destination Marketing \& Management, 1 (1-2), 47-55, doi:10.1016/j.jdmm.2012.09.003.

International Snowmobile Manufacturers Association, 2014: Snowmobiling fact book. 37 pp. [Available online at www.snowmobile. org/docs/snowmobiling-fact-book-2013-2014.pdf.]

Jack, S. J., and K. R. Ronan, 1998: Sensation seeking among highand low-risk sports participants. Pers. Individ. Differ., 25, 1063-1083, doi:10.1016/S0191-8869(98)00081-6.

Katz, R. W., and A. H. Murphy, 1997: Economic Value of Weather and Climate Forecasts. Cambridge University Press, 222 pp.

Kilpeläinen, M., and H. Summala, 2007: Effects of weather and weather forecasts on driver behaviour. Transp. Res. Part F, 10, 288-299, doi:10.1016/j.trf.2006.11.002.

Knapp, K., 2001: Investigation of volume, safety, and vehicle speeds during winter storm events. Proc., Ninth Maintenance Management Conf., Juneau, AK, Transportation Research Board, 57-64.

Lazo, J. K., and L. G. Chestnut, 2002: Economic value of current and improved weather forecasts in the U.S. household sector. Rep. to the NOAA Office of Policy and Planning, 213 pp. [Available online at http://www.wmo.int/pages/prog/amp/pwsp/ documents/STORM_Report.pdf.]

— , R. E. Morss, and J. L. Demuth, 2009: 300 billion served: Sources, perceptions, uses and values of weather forecasts. Bull. Amer. Meteor. Soc., 90, 785-798, doi:10.1175/2008BAMS2604.1.

Maunder, W. J., 1969: The consumer and the weather forecast. Atmosphere, 7, 15-22.

Morss, R. E., J. K. Lazo, B. G. Brown, H. E. Brooks, P. T. Ganderton, and B. N. Mills, 2008: Societal and economic research and applications priorities for the North American THORPEX program. Bull. Amer. Meteor. Soc., 89, 335-346, doi:10.1175/ BAMS-89-3-335.

- - and J. L. Demuth, 2010: Examining the use of weather forecasts in decision scenarios: Results from a U.S. survey with implications for uncertainty communication. Meteor. Appl., 17, 149-162, doi:10.1002/met.196.

National Research Council, 2006: Completing the Forecast: Characterizing and Communicating Uncertainty for Better Decisions Using Weather and Climate Forecasts. National Academies Press, 124 pp.
- 2010: When Weather Matters: Science and Service to Meet Critical Societal Needs. National Academies Press, 208 pp.

National Ski Areas Association, 2013: 2011-12 economic analysis of U.S. ski areas. NSAA \& RRC Associates, 107 pp.

National Weather Service, 2010: NOAA's National Weather Service strategic plan: 2011-2020. NOAA, 20 pp. [Available online at http://www.nws.noaa.gov/com/stratplan/files/plan_v01.pdf.]

OECD, cited 2013: Glossary of statistical terms. [Available online at http://stats.oecd.org/glossary/detail.asp?ID =6185.]

Ontario Federation of Snowmobile Clubs, 2013: Annual report 2012. OFSC, $20 \mathrm{pp}$.

Ontario Ministry of Tourism, Culture and Sport, 2011: Concepts and definitions. Tourism Research Unit, 7 pp. [Available online at www.mtc.gov.on.ca/en/research/resources/Concepts $\% 20$ Definitions.pdf.]

Ontario Snow Resorts Association, 2013: OSRA 2011-2012 end of season report. OSRA, $22 \mathrm{pp}$.

Pew Research Center, 2008: Audience segments in a changing news environment: Key news audiences now blend online and traditional sources. Pew Research Center Biennial News Consumption Survey, 129 pp. [Available online at http:// people-press.org/files/legacy-pdf/444.pdf.]

Rutty, M., and D. Scott, 2010: Will the Mediterranean become "too hot" for tourism? A reassessment. Tourism Hospitality Plann. Dev., 7, 267-281, doi:10.1080/1479053X.2010.502386.

Scott, D., and C. Lemieux, 2010: Weather and climate information for tourism. Procedia Environ. Sci., 1, 146-183, doi:10.1016/ j.proenv.2010.09.011.

,-- , and L. Malone, 2011: Climate services to support sustainable tourism and adaptation to climate change. Climate Res., 47, 111-122, doi:10.3354/cr00952.

Smith, K., 1993: The influence of weather and climate on recreation and tourism. Weather, 48, 398-404, doi:10.1002/ j.1477-8696.1993.tb05828.x.

Statistics Canada, cited 2013: Amusement and recreation, summary statistics. [Available online at www.statcan.gc.ca/tablestableaux/sum-som/101/cst01/arts60a-eng.htm.]

Stewart, A. E., J. K. Lazo, R. E. Morss, and J. L. Demuth, 2012: The relationship of weather salience with the perceptions and uses of weather information in a nationwide sample of the United States. Wea. Climate Soc., 4, 172-189, doi:10.1175/WCAS-D-11-00033.1.

Stewart, T. R., R. Pielke, and R. Nath, 2004: Understanding user decision making and the value of improved precipitation forecasts: Lessons from a case study. Bull. Amer. Meteor. Soc., 85, 223-235, doi:10.1175/BAMS-85-2-223.

Unrau, D., and J. Andrey, 2006: Driver response to rainfall on urban expressways. Transp. Res. Rec., 1980, 24-30, doi:10.3141/ 1980-06

UNWTO, cited 2013: Understanding tourism: Basic glossary. [Available online at http://media.unwto.org/en/content/understandingtourism-basic-glossary.]

Wilson, K., 2008: Television weathercasters as science communicators. Public Understanding Sci., 17, 73-98, doi:10.1177/ 0963662506065557.

World Meteorological Organization, cited 2013: World Climate Programme. [Available online at www.wmo.int/pages/prog/ wcp/wcp.html.]

Zuckerman, M., 1979: Sensation Seeking: Beyond the Optimal Level of Arousal. Lawrence Erlbaum Associates, $464 \mathrm{pp}$.

- 1983: Sensation seeking and sports. Pers. Individ. Differ., 4, 285-293, doi:10.1016/0191-8869(83)90150-2. 